\title{
Social and Private Learning with Endogenous Decision Timing
}

\section{Julian Jamison, David Owens, and Glenn Woroch}

\begin{abstract}
Firms often face choices about when to upgrade and what to upgrade to. We discuss this in the context of upgrading to a new technology (for example, a new computer system), but it applies equally to the upgrading of processes (for example, a new organizational structure) or to individual choices (for example, buying a new car). This paper uses an experimental approach to determine how people address such problems, with a particular focus on the impact of information flows. Specifically, subjects face a multi-round decision, choosing when (if ever) to upgrade from the status quo to either a safe or a risky new technology. The safe technology yields more than the status quo, and the risky technology may yield either less than the status quo or more than the safe technology. Every round, subjects who have not yet upgraded receive noisy information about the true quality of the risky technology. Our focus on the timing of endogenous choice is novel and differentiates the results from previous experimental papers on herding and cascades. We find that, in the single-person decision problem, subjects tend to wait too long before choosing (relative to optimal behavior). In the second treatment, they observe payoff-irrelevant choices of other subjects. This turns out to induce slightly faster decisions, so the "irrationality" of fads actually improves profits in our framework. In the third and final treatment, subjects observe payoff-relevant choices of other subjects (that is, others who have the same value for the risky technology but independent private signals). Behavior here is very similar to the second treatment, so having "real" information does not seem to have a strong marginal effect. Overall we find that social learning, whether or not the behavior of others is truly informative, plays a large role in upgrade decisions and hence in technology diffusion.
\end{abstract}

\section{JEL codes: L15, C91, D83}

Julian Jamison is a senior economist at the Federal Reserve Bank of Boston's Center for Behavioral Economics and Decisionmaking, David Owens is an assistant professor of economics at Haverford College, and Glenn Woroch is an adjunct professor of economics at the University of California, Berkeley. Their e-mail addresses are julian.jamison@bos.frb.org, dowens@haverford.edu, and glenn@econ.berkeley.edu, respectively.

This paper, which may be revised, is available on the web site of the Federal Reserve Bank of Boston at http://www.bos.frb.org/economic/wp/index.htm.

We thank audiences at the French Economic Association (Lyon), Industrial Organization Meeting (Lecce), and Experimental Game Theory (Florida State), as well as France Telecom Research \& Development for financial support.

The views expressed in this paper are solely those of the authors and not those of the Federal Reserve System or the Federal Reserve Bank of Boston.

This version: September 9, 2009

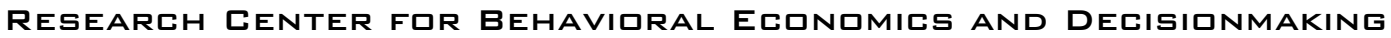




\section{Introduction}

Individuals and organizations routinely have the option to undertake large, sunk investments in technologies that could drastically alter how they operate. Typically, these expenditures come with significant risk: besides uncertainty as to whether a new technology will live up to its promises, the return on investment depends on factors outside their control such as cost of complements, market conditions, and macroeconomic trends. Present day examples include the deployment of an advanced computing or communications system or the adoption of green technologies to conserve energy and reduce pollution. While adoption initiates the stream of benefits from the innovation - whether those take the form of lowered costs or a new revenue source - delay allows a firm to gather additional information on the prospects of the technology's profitability.

This paper employs laboratory experiments to investigate patterns of behavior that govern firm and industry adoption of innovations as they balance the tension between acting quickly and waiting for more information. In our experiments, subjects choose between a safe and a risky innovation and decide when to undertake the adoption as well. Prior to adoption, subjects earn a return associated with a status quo technology that is smaller than the return on the safe innovation. We implemented three treatments that are nested in terms of the amount of information made available to the subjects. In addition to knowledge of the risk and return properties of the three technologies, each subject observes a private, informative signal of the true return of the risky innovation, and in two of the three treatments, the prior adoption decisions of other subjects as well. Two of our treatments assign "private values" for the outcome of the risky innovation to subjects, while they have a "common value" in the third treatment, borrowing terminology from auction design. Adoptions are irreversible, so delay is the only means to acquire this additional information. Delay is not costless, however, since subjects incur an opportunity cost equal to the difference between the per-period profitability of the safe innovation and the status quo technology.

We are particularly interested in the relationships among the flows of information, in the choice between the safe and risky innovation, and in the timing of the adoption. In particular, to what extent do subjects delay adoption to gather private and public information, and do the two sources of information impact timing in different ways?

The laboratory experiments offer tests of several behavioral hypotheses. From 
a purely decision-theoretic perspective, subjects may rely solely on their private information to decide whether to opt for the risky alternative, ignoring completely the choices made by others. Delay would derive from the firm's desire to gather more private information to make a better choice between the two investment alternatives.

At the other extreme, subjects may simply ignore their private information and imitate the adoption decisions of others who acted earlier. While such unreflective imitation can accelerate the diffusion of an innovation through the population, it can also lead to industry-wide selection of an inferior technology. Furthermore, such conformity could also create perverse incentives, as when a firm adopts early to steer the industry toward one technology rather than another.

This paper contributes to the literature on the causes and patterns of the adoption and diffusion of innovations. In this vast literature, we seek to contribute to the portion that deals with how information is used by potential adopters to select among available innovations and to decide when to adopt them. As Geroski (2000) notes in his survey of the economics of diffusion, economists are generally puzzled how, in practice, superior technologies diffuse slowly through the population. Indeed, many of the contributions to this literature propose causes for the observed delay in adopting improvements over the status quo. We are particularly interested in the diffusion research that investigates whether all the information available to members of the industry is employed in making the choice among several potential innovations. In particular, is the best technology chosen given the available information?

Various answers have been offered to these questions depending on the modeling approach taken. Perhaps the best known among these is the "epidemic model," which assumes growth in penetration of a new technology is proportional to the size of the population that has already adopted. The narrative that accompanies this specification claims that potential adopters who have not yet adopted have an equal chance of encountering each individual in the population, and when they encounter an adopter, there is a given chance they will follow suit and adopt the innovation. Adopters, in effect, communicate or infect some fraction of the non-adopters in each period. The penetration of the innovation grows according to a familiar S-shaped curve.

Griliches (1957) and Mansfield (1961) conducted some of the earliest econometric estimates of the diffusion of process innovations. Importantly, they focused attention on the role of firms' specific conditions in determining the diffusion of 
innovations over time. Rogers (1962) focused, instead, on how communication between adopters and non-adopters caused the spread of innovations, with particular emphasis on the heterogeneity across potential adopters in their receptiveness to new technology. ${ }^{1}$

A second model of innovation diffusion - one that Geroski (2000) refers to as the "probit model" - relies on heterogeneity among firms to explain the timing of adoptions. Differences in firms' costs, demands, or resources lead to differing optimal dates for adoption of an innovation, and collectively lead to gradual diffusion of the innovation as the cost of adoption decreases. Our experimental design precludes communication among subjects, except when prior adoption decisions are publicly reported. It also suppresses any heterogeneity among subjects, aside from the unobserved characteristics of the individuals selected to participate in our experiments. The purpose of this design is to isolate the role of information flows as the cause of adoption decisions. In addition, subjects earn rewards that are independent of any other subject's action. We designed the experiments so as to avoid "payoff externalities," as they can cause herding behavior among subjects, which could be difficult to separate from that caused by "informational externalities."

Subjects' decision problems in our experiments resemble a third class of adoption models in which profitability of innovations is uncertain and private signals are observed that can reduce that uncertainty. Jensen $(1982,1988)$ generates gradual diffusion of an innovation because firms possess different amounts of information about the uncertain profitability of the innovation. Firms reduce this uncertainty by delaying adoption and acquiring private signals. In that case, firms either adopt immediately if they are sufficiently optimistic about the technology's prospects, or delay for an uncertain number of periods until they reach a minimum level of confidence about its profitability.

While adopter heterogeneity is again the cause of diffusion in this class of models, it derives from the different sequences of signals drawn by the firms. In contrast, Kapur (1995) generates diffusion without private signals, but by having firms observe adoption decisions of other firms in the industry. Firms then enter into "waiting contests" as each one prefers to free ride on the information generated by earlier adopters.

\footnotetext{
${ }^{1}$ Ellison and Fudenberg (1993) provide a formal analysis of some of the diffusion dynamics that were discussed by Rogers. Their model allows for an additional source of learning that occurs when potential adopters can observe the consequences of adoptions made by their neighbors, as well as which technology they chose. The subjects in our experiments, on the other hand, do not observe the payoffs earned by their peers.
} 
While many econometric studies of diffusion models have been undertaken, few laboratory experiments have been conducted to test various hypotheses. Among the exceptions are the papers by Isaac and Reynolds (1988, 1992) and Zizzo (2002) that test several theories of $\mathrm{R} \& \mathrm{D}$ competition and patent races in a laboratory setting. In another laboratory experiment narrowly focused on the impacts of subsidies, Aalbers et al. (2007) examine adoption behavior of professional managers. These papers, along with the bulk of the econometric literature on adoption and diffusion of innovation, take into account the connection between the profitability of the innovation and its impact on product market competition. In particular, profitability of the innovation depends on the firm's position in the order of adoption and on the structure of the product industry. In our treatments, we simplify the experimental task for our subjects by suppressing any such interaction. And while we recognize that downstream competition among potential adopters is a critical feature of the adoption decision, as are any other "payoff externalities" that could arise among subjects, we choose to examine those aspects separate from the potential for "information externalities."

Our experimental design, together with the hypotheses about adoption behavior, has been greatly influences by the rapidly growing body of research on social learning games and experiments. Theoretical models in that literature analyze sequential investment games played by rational agents who have access to both private and public information. These models have been preoccupied with the possibility that adopters choose to imitate prior adoptions as a means to free ride on the information gathered by others. Banerjee (1992) and Bikhchandani, Hirshleifer, and Welch (1992) find Bayesian-Nash equilibria in which potential adopters choose to ignore their private signals and simply imitate adoption decisions of predecessors. In those papers, agents have the opportunity to act at a single time in a pre-determined order. Chamley and Gale (1994) and Chamley (2004) consider equilibrium of a private-signal, common-value game in which players are free to choose the timing of a binary investment. They find that, in Bayesian equilibrium, players delay their choice, and that the extent of delay is directly related to the duration of a period and inversely related to the number of players. Zhang (1997) also allows for endogenous timing in a binary investment game in which time is continuous. He finds that, in equilibrium, agents engage in delay to learn from others, but once investment begins to occur, subsequent investments occur rapidly.

A number of laboratory experiments have sought to gauge the incidence of in- 
formation cascades and herding behavior. Anderson and Holt (1997) was the first in a series of laboratory experiments to test the theoretical predictions of information cascades. Designing experiments that adhere closely to the fixed timing setup found in Bikhchandani et al. (1992), they find that their subjects demonstrate a high level of agreement with theoretical predictions. For instance, subjects fail to make their choice between two investments either on their private information or on Bayesian updating of observed prior adoptions in a small percentage (4 percent) of cases. Less than a third (31.7 percent) of cascades were on the wrong investment, and infrequently (26 percent) subjects would follow their private signal when they should have followed prior adoptions.

A series of laboratory experiments followed Anderson and Holt (1997); they differ in minor ways from their original setup, and yielded more or less the same results. Of particular importance to this study, all of these experiments imposed a pre-determined order on subject decision-making, as specified in the Bikhchandani et al. (1992) model. One exception is Sgroi (2003), who allowed subjects to choose the timing of their adoption. He found that subjects would, indeed, delay adoption to learn about others' private information, but that herding behavior persisted; in fact, reverse cascades appeared to become more numerous. Celen and Kariv (2004a) were the first to distinguish between herd behavior and information cascades in a laboratory setting. Drehmann, Oechssler, and Roider (2007) conducted online experiments in which subjects also chose the timing of their investments. Compared with a dozen articles implementing the structure of Bikhchandani et al. (1992), their online subjects rely on the private signals to a much greater extent. They also follow their private signal when they should follow prior adoptions in a larger portion of the cases (34 percent) than in the experimental results.

In our laboratory experiments, subjects can choose at any time a safe innovative technology $(B)$, or a risky innovative technology $(C)$ having the same expected payoff. They earn a lower return from a status quo technology $(A)$ each round that they delay before adopting. Treatment $\mathrm{P}$ is based on private values because the outcome of the risky innovation is randomly assigned across the subjects. Subjects receive a noisy signal about its true value at the beginning of the eight rounds. Treatment $P O$ is identical to treatment $P$ except that subjects also learn the total number of $B$ and $C$ adoptions up to the current round. Since the private value structure is maintained, this adoption information should have no value for subjects. Treatment $C O$, on the other hand, begins by drawing a value for the 
risky innovation that is common for all subjects, in which case public knowledge of prior adoptions should be informative.

To establish a benchmark against which to evaluate the experimental results, we solve for the Bayesian-Nash equilibrium strategies for the subjects in each of the three treatments. Using backward induction, we should expect that riskneutral payoff maximizers will adopt one of the two innovations in the first period according to which one is favored by their first signal. This solution applies to treatments $P$ and $P O$, since observation of prior adoptions contains no payoffrelevant information. Turning to treatment $C O$ where payoff from $C$ is perfectly correlated across subjects, we do not characterize all the Bayesian-Nash equilibria because of the size of the state space of the game. We do expect, however, that subjects will conform to this equilibrium prediction by adopting the innovation indicated by their first signal. As a result, equilibrium behavior will have some subjects delay adoption and observe the behavior of others.

In the discussion of our results, we focus on the (sometimes) competing influences of private and social learning. Private learning is the influence that a subject's private information has on her decisions. Social learning refers to the influence that the observation of others' actions has on decisions. In treatment $P O$, subjects have the opportunity to be influenced by others, although this observation is not statistically relevant. We categorize such an influence as "social learning," and reserve the term "Bayesian learning" for the influence in treatment $C O$, where Bayesian updating can take place as a result of this observation.

The results of the three treatments reveal preferences for adopting one innovation or another, for the timing of the adoption decisions, and for the overall profits earned by the subjects. On average, subjects show a slight preference for the safe innovation $(B)$ over the risky one $(C)$. Their adoption decisions significantly improve upon pure randomization, indicating that subjects incorporate private and public information into their decision-making. Surprisingly, they do a better job at picking the better of the two innovations when they receive noninformative reports on prior adoptions in treatment $P O$ than when those reports contain valuable information as in the case of treatment $C O$.

Turning to the timing of adoptions, roughly half of subjects in all treatments do not adopt in the first round as prescribed by the equilibrium for $P$ and $P O$. One interpretation of this departure from equilibrium is that subjects seek to learn from others by delaying. Interestingly, when subjects observe their peers, that is, in treatments $P O$ and $C O$, they adopt more quickly as a group. This 
suggests that early adopters apply "competitive pressure" on other subjects to act, even when that action leads to an adoption different from the most popular prior adoption. Profits earned by subjects appear to be related to their access to information, in that subjects earn a higher profit when they have the opportunity to observe their peers. This is, in large part, due to the competitive pressure explained above.

The next section provides more details about our three experimental treatments. The subsequent section solves the formal decision problems for each of these three treatments as if the subjects were perfectly rational. We then report the experimental results comparing the three treatments against one another and against our theoretical predictions. We close the paper with a summary of our conclusions.

\section{Experimental Design and Procedures}

All experiments were conducted using Experimental Social Science Laboratory, or "Xlab," at the University of California, Berkeley. The Xlab primarily recruits undergraduate students as subjects, with some graduate students and university staff members as well.

The experiment itself was conducted on laptop personal computers implementing a z-Tree (Zurich Toolbox for Readymade Economic Experiments) program. Workstations were separated from one another with physical partitions to preclude subjects from looking at one another's screens, and to discourage conversation. Further, apart from the introductory period when subjects could ask clarifying questions, no oral communication was allowed. Subjects participated in just one experimental session and, hence, in just one treatment.

Subjects were offered monetary incentives for their participation in the experiments. These were denominated in tokens during the experiment, and exchanged at a rate told to subjects at the beginning of each session. ${ }^{2}$ When all subjects had completed the experiment, payoffs were determined and individual checks were issued in sealed envelopes. The average payout per subject per session was $\$ 27.32$.

Our experiments consist of seven different sessions. On average, 16.4 subjects participated in each experimental session. Upon arriving, subjects were given

\footnotetext{
${ }^{2}$ The exchange rate was eight tokens per dollar in treatment $P$ and twelve tokens per dollar in treatments $P O$ and $C O$.
} 
written instructions and ten minutes to read them. The instructions were then read aloud by a researcher. Subjects were randomly assigned to groups, each consisting of six members. ${ }^{3}$ They were not informed of the identities of the other members of their group. In most cases, the subjects were asked to take a simple quiz that tested their understanding of the rules before beginning the experiment. They were not allowed to proceed until they answered the questions correctly. ${ }^{4}$

In each treatment, subjects participated in a series of 16 decision problems, each of which consisted of eight rounds. The first of the 16 decision problems was unpaid practice and the results were discarded, leaving data for 15 rounds per subject. The number of rounds, decision problems, and size of groups were fixed throughout all of the experimental treatments and sessions in order to maintain comparability.

Across treatments, each subject's task is to adopt one of two technologies, $B$ or $C$. As previously stated, they also decide when to adopt. Before they adopt, they are on the inferior "status quo," technology A. Each technology has a "value" associated with it, which is a per-round payoff that accrues to each subject on that technology. Technologies $A$ and $B$ had a value of 1 and 2 tokens per round, respectively. Technology $C$ 's value was stochastic, realizing values of 0 and 4 with equal probability. We follow the classic ball and urn design of Anderson and Holt (1997) in which subjects receive an informative but noisy private signal about the realized value of technology $C$ before they make their decision for round 1. Each subject who declines to adopt technology $B$ or $C$ in round 1 remains active, and receives further information about her decision.

Active subjects begin each round of a decision problem by observing a private signal. If the value of technology $C$ is 0 , the subject receives a draw from an "L-urn" (as in "low" payoff). That urn generates an " $H$ " signal with a probability of $\frac{1}{3}$ and an " $L$ " signal with a probability of $\frac{2}{3}$. If technology $C$ 's value is 4 , then the signal is drawn from the H-urn, reversing the conditional probabilities of the two signals: " $H$ " arises with probability $\frac{2}{3}$ and "L" with probability $\frac{1}{3}$. As technology $C$ 's value can be low or high with equal probability probability, its conditional probability distribution given that the "L" signal is observed is $\frac{2}{3}$ and $\frac{1}{3}$, respectively, for values of 0 tokens and 4 tokens.

In each round, active subjects make decisions among the technologies $A, B$, and $C$. The option to choose the timing of one's adoption decision sets our exper-

\footnotetext{
${ }^{3}$ This was not the case in treatment $P$, where there were no groups.

${ }^{4}$ This was done in all but the first round of sessions. No difference was found after adding the quiz.
} 
iment apart from the others. Most experimental studies of technology adoption follow the traditional modeling of herding in which a subject occupies a predetermined position in a sequence of decisions and has only one opportunity to make a decision. We do preserve one property of that tradition, however, when we assume that the choice of either $B$ or $C$ is irreversible. Once a subject adopts either technology, she remains with that technology for the remainder of the decision problem. If, instead, she remains with technology $A$, she retains the option to adopt $B$ or $C$, or to continue with technology $A$ in the next round. For each round that a subject chooses technology $A$, she draws a new signal from the corresponding urn. In this way, technology $A$ is the status quo that allows the subject to acquire additional information about technology $C$.

The value of this additional information, even in theoretical terms, depends on the experimental structure of the various treatments that we implement. Besides an additional draw of the private signal, in most treatments subjects can observe their peers' adoption decisions. It is through this channel that social learning, if it occurs, takes place.

\subsection{The Three Treatments}

We ran three different treatments over the course of our seven experimental sessions. They were nested to identify the effects of private learning and social learning - both Bayesian and non-Bayesian.

\section{- Treatment $P$ : Private Values, No Observation}

In Treatment $P$, technology $C$ 's value is independently determined for each subject, and there is no observation. Subjects simply observe their private signals and never observe what choices other subjects make, much less when they make them. This is our baseline experiment as only private learning, and no social learning, is possible.

\section{- Treatment $P O:$ Private Values, Observation}

This treatment employs a payoff structure identical to that of treatment $P$. Technology $C$ 's value is determined independently for each subject. However, beginning in round 2 of each decision problem, subjects learn how many members have, as of yet, adopted technologies $B$ and $C$. Technology 
$C$ 's value is completely uncorrelated across subjects, so Bayesian learning is impossible.

\section{- Treatment $C O$ : Common Values, Observation}

From the subjects' perspective, treatment $C O$ appears identical to treatment $P O$. However, payoffs are perfectly correlated within groups: technology $C$ 's value is the same for all members of each group ( 0 or 4 , each with equal probability). This "common value" creates an opportunity for Bayesian learning from prior actions of others.

Treatment $P$ serves as a baseline, and explores the private learning process. As there is no social interaction in this treatment, there can be no social learning. In treatment $P O$, there is no Bayesian learning. Still, subjects observe the decisions of five other subjects. If behavior in treatment $P O$ differs from that in treatment $P$, social learning is a possible explanation, while Bayesian learning is not. In Treatment $C O$, technology $C$ 's value is the same for all members of the group. As subjects can update their beliefs on the value of technology $\mathrm{C}$ by observing the choices of other members of her group, Bayesian learning is possible.

\section{Theory}

This section derives the optimal decision rule for subjects who participate in each of the three experimental treatments. We assume that subjects are risk-neutral, expected-utility maximizers who obey the basic laws of probability. Accordingly, subjects will adopt the technology that maximizes their expected payoff, equal to the (undiscounted) sum of the tokens they earn over the entire decision problem.

Before formalizing the subject's decision problem, note that three events occur within each of the eight rounds. At the beginning of round $t$, all active subjects draw a private signal of the state of nature of technology $C$. Given the outcome of that draw and all previous draws, and given observed adoptions of all other subjects up through round $t-1$ in the case of treatments $P O$ and $C O$, each subject chooses from among the three technologies, where choice of status quo technology $A$ is equivalent to postponing the decision between $B$ and $C$ for at least one more round. At the end of the round, payoffs are realized according to which technology was chosen in the round, although tokens are not awarded until the end of the decision sequence. 
We need some notation to begin with. Let $V_{j}(h, t)$ denote the expected value of technology $j \in\{A, B, C\}$ earned by a subject in round $t$ when she has observed $h$ private signals to be $H$ up through and including round $t$. Then $\pi_{j}(h, t)=$ $V_{j}(h, t) \times(9-t)$ gives the total expected profit from technology $j$ over the remainder of the decision problem if technology $j$ is chosen in that round. Finally, let $X^{*}(h, t)$ be a subject's set of optimal choices, that is, $j \in X^{*}(h, t)$ if $\pi_{j} \geq \pi_{k} \forall k \in\{A, B, C\}$.

For treatments $P$ and $P O$, the solution is an optimal decision rule that maps their information sets into a probability distribution over the three technologies. Because in treatment $C O$ a subject's optimal action may also depend on the observed adoptions made by others, subjects' payoffs become interdependent. ${ }^{5}$ As a consequence, we must look for equilibrium noncooperative strategies for a subject. Of course, subjects could also condition their adoptions on these observed adoptions in treatment $P O$, but, in theory, it would not offer any monetary benefit to subjects.

\subsection{Treatments $P \& P O$}

Since treatments $P$ and $P O$ pose decision problems that are technically equivalent, we characterize a single optimal decision rule for both in this section.

If the timing of decisions were exogenous, ruling out delay as an option, then the optimal decision rule would be quite simple. Subjects would simply adopt technology $B$ when $h<l$, where $l$ is the cumulative number of $L$ draws, and they would adopt technology $C$ when $h>l$.

The opportunity to delay by remaining with the status quo technology $A$ complicates the characterization of the optimal decision rule. The calculations of expected payoffs for technologies $B$ and $C$ are quite simple: $V_{B}=2$ and $V_{C}(h, t)=4 \times P_{4}(h, t)$, where $P_{4}(h, t)$ denotes the probability that $V_{C}=4$ given $h$ and $t$. As the prior is $50: 50$ on the two states of nature, the posterior simplifies to:

$$
P_{4}(h, t)=\frac{2^{h}}{2^{h}+2^{t-h}} .
$$

The probability that technology $C$ 's value is zero is $P_{0}(h, t)=1-P_{4}(h, t)$. Substituting $l=t-h$, the cumulative number of $L$ signals received, into equation 1 , and simplifying, yields

$$
P_{4}(h, t)=\frac{2^{h-l}}{2^{h-l}+1} .
$$

\footnotetext{
${ }^{5}$ Though payoffs remain independent of other subjects' actions
} 
Equation 2 reveals a key insight. $P_{4}(h, t)$ depends only on $(h-l)$. In other words, the likelihood of $C$ having the high-value realization 4 depends only on how many more $H$ draws than $L$ draws have been observed, and not on the totals: $P_{4}(1,1)=P_{4}(4,7)$, for example. Also note that we implicitly assume that subjects' perception of probability is not affected by the order of the signals, but only by their cumulative number.

$V_{A}(h, t)$ is more difficult to compute. Given $(h, t)$, we must consider the probability of receiving a private signal of $H$ or of $L$ in round $t+1$, and multiply these by $X^{*}(h+1, t+1)$ and $X^{*}(h, t+1)$, respectively. One must also consider the fact that the optimal action in the next period may be to again remain with technology $A$.

As continuation strategies depend on future private signals, it is necessary to compute $P_{H}(h, t)$, the probability that the next private signal is $H$. Recall that such a signal may come from either realization for $C$ 's value, 0 or 4 :

$$
P_{H}(h, t)=\frac{2}{3} \times P_{4}+\frac{1}{3} \times P_{0} .
$$

In any round, $\pi_{A}$ is the value of $A$ (which is 1 ) plus the expected value of the best choice in the next round, given a signal of $H$ or $L$, times $(8-t)$, the number of rounds remaining after a decision in round $t+1$ :

$$
\pi_{A}(h, t)=1+\left[P_{H} \times \pi_{M}(h+1, t+1)+\left(1-P_{H}\right) \times \pi_{M}(h, t+1)\right] \times(8-t),
$$

where

$$
\pi_{M}(h, t)=\max \left\{\pi_{A}(h, t), \pi_{B}(h, t), \pi_{C}(h, t)\right\},
$$

that is, $\pi$ evaluated for $X^{*}(h, t)$.

Of course, in round $t+1$, it may again be optimal choice to delay by choosing the status quo technology $A$, and then again in round $t+2$, and so on. Due to this complication, and the finite number of rounds in our setup, we solve the decision problem using backward induction, starting with the last round where subjects' optimal decisions are obvious.

A large number of continuation strategies need to be examined to construct the optimal decision rule, but a few observations greatly reduce the number of candidates. Note, first, that opting for the status quo technology $A$ amounts to 
purchasing information about the value of the risky technology, $C$. Eventually, all subjects adopt either $B$ or $C$. Therefore, if any subject knows in round $t$ that she will adopt technology $B$ or $C$ in round $t+1$, then she should make that adoption in round $t$. A subject should delay choosing $B$ or $C$ only if the next signal has the potential to change her decision.

Second, the amount of information acquired by delay is more costly if $h>l$, because of the asymmetry in uncertainty between $B$ and $C$. If $h>l$, then $V_{C}>V_{B}=2$, that is, if a subject were forced to choose between $B$ and $C$, she should opt for $C$ since $\max \left\{V_{B}, V_{C}\right\}>2$. If $h \leq l$, on the other hand, then $\max \left\{V_{B}, V_{C}\right\}=2$. Therefore, for $h>l, V_{C}(h, t)-V_{A}(h, t)>V_{B}(l, t)-V_{A}(l, t)$. In other words, because the opportunity cost of remaining with technology $A$ is higher when $h>l$, information will be more valuable when $h<l$. As a consequence:

$$
\begin{aligned}
& \pi_{B}(h, t)>\pi_{A}(h, t) \Rightarrow \pi_{C}(t-h, t)>\pi_{A}(t-h, t) \\
& \text { and } \\
& \pi_{A}(h, t)>\pi_{C}(h, t) \Rightarrow \pi_{A}(t-h, t)>\pi_{B}(t-h, t) .
\end{aligned}
$$

Relation 6 means that, for a given $l-h$, if subjects prefer adopting technology $B$ to remaining with technology $A$, they would prefer adoption of technology $C$ to remaining with $A$ if $h-l$ took on the same value. Further, for a given $h-l$, if subjects prefer remaining with technology $A$ to adopting technology $C$, then they prefer $A$ over $B$ for $l-h$ of the same value.

An active subject in round 8 has no reason to choose technology $A$ because delay is no longer possible. Hence,

$$
X^{*}(h, 8)= \begin{cases}B & \text { for } h<4 \\ \{B, C\} & \text { for } h=4 \\ C & \text { for } h>4\end{cases}
$$

As early adoption dominates later adoption, we know that a subject should adopt technology $C$ as soon as $h=4$, and technology $B$ as soon as $l=4$, whenever those events occur. This also makes any decision in round 7 trivial, 
since after the draw in that round either $l \geq 4$ or $h \geq 4$. Therefore,

$$
X^{*}(h, 7)=\left\{\begin{array}{l}
B \text { for } h<4 \\
C \text { for } h \geq 4
\end{array} .\right.
$$

The decision in round 6 is trivial for $h<3$ and $h>3$, but the case for $h=3$ is not as simple. For $h=3, V_{B}=V_{C}=2$. Remaining with technology $A$ would allow a subject to make a more informed decision between technologies $B$ and $C$ in round 7 (as shown above, subjects will adopt in round 7 no matter what). The drawback is an additional round of earning only $A$ 's value of 1 . She will adopt technology $B$ if she receives one more $L$ signal, and technology $C$ if she receives one more $H$ signal. Thus, $\pi_{A}(6,3)$ is 1 plus the probability of $L$ times $\pi_{B}(3,7)$ plus the probability of $H$ times $\pi_{C}(4,7)$, or (using the fact that the current symmetry implies equal probabilities for $H$ and $L$ signals):

$$
\begin{aligned}
\pi_{A}(3,6) & =1+P_{H} \times \pi_{B}(3,7)+\left(1-P_{H}\right) \times \pi_{C}(4,7) \\
& =1+\left[\frac{1}{2} \times \frac{2^{1}}{2^{1}+1} \times 4+\frac{1}{2} \times 2\right] \times(8-6)=\frac{17}{3} . \\
\pi_{B}(3,6) & =2 \times(9-6)=6 . \\
\pi_{C}(3,6) & =\frac{1}{2} \times 4 \times(9-6)=6 .
\end{aligned}
$$

Thus,

$$
X^{*}(h, 6)= \begin{cases}B & \text { for } h<3 \\ \{B, C\} & \text { for } h=3 \\ C & \text { for } h>3\end{cases}
$$

Similar to round 7, equation 9 renders the analysis for round 5 trivial:

$$
X^{*}(h, 5)=\left\{\begin{array}{l}
B \text { for } h<3 \\
C \text { for } h \geq 3
\end{array} .\right.
$$

In round 4 , subjects should clearly adopt $B$ if $h<2$, and $C$ if $h>2$.

We now need to determine $X^{*}(2,4)$. The tradeoff is very similar to that when 
$h=3$ and $t=6$, but more rounds remain in the future:

$$
\begin{aligned}
\pi_{A}(2,4) & =1+P_{H} \times \pi_{C}(3,5)+\left(1-P_{H}\right) \times \pi_{B}(2,5) \\
& =1+\left[\frac{1}{2} \times \frac{2^{1}}{2^{1}+1} \times 4+\frac{1}{2} \times 2\right] \times(8-4)=\frac{31}{3} . \\
\pi_{B}(2,4) & =\pi_{C}(2,4)=10 .
\end{aligned}
$$

So,

$$
X^{*}(h, 4)=\left\{\begin{array}{l}
B \text { for } h<2 \\
A \text { for } h=2 \\
C \text { for } h>2
\end{array}\right.
$$

As $\pi_{A}(2,4)>\pi_{B}(2,4)=\pi_{C}(2,4)$, subjects benefit from remaining with technology $A$ when $h=2$ and $t=4$. Compared with round 6 , there are more rounds in the future to reap the benefit of the additional information, and hence the additional information becomes worth the opportunity cost (of 1 token per round).

Given that $X^{*}(2,4)=A$, equilibrium predictions for round 3 behavior are less trivial than for rounds 5 and 7 . As $X^{*}(1,4)=B$ and $X^{*}(3,4)=C$, we also know that $X^{*}(0,3)=B$ and $X^{*}(3,3)=C$. We must find $X^{*}(1,3)$ and $X^{*}(2,3)$. Statement 6 shows us that if $X^{*}(1,3)=B$, then $X^{*}(2,3)=C$. Therefore, we compare $\pi_{A}(1,3)$ to $\pi_{B}(1,3)$ :

$$
\begin{aligned}
\pi_{A}(1,3) & =1+P_{H} \times \pi_{A}(2,4)+\left(1-P_{H}\right) \times \pi_{B}(1,4) \\
& =1+\frac{4}{9} \times \frac{31}{3}+\frac{5}{9} \times 2 \times(8-3)=\frac{301}{27} \approx 11.15 \\
\pi_{B}(1,3) & =2 \times(9-3)=12 .
\end{aligned}
$$

Here we have used $P_{H}(h, t)=\frac{2}{3} \times P_{4}+\frac{1}{3} \times P_{0}$ (as stated above), along with $P_{4}=\frac{2}{2+4}$, and likewise for $P_{L}$.

As $\pi_{B}(1,3)>\pi_{A}(1,3), X^{*}(1,3)=B$. By statement $6, X^{*}(2,3)=C$. Intuitively, the opportunity cost of waiting is higher when $h>l$, as the choice of option $A$ forgoes a higher expected value in that case.

$$
X^{*}(h, 3)=\left\{\begin{array}{l}
B \text { for } h<2 \\
C \text { for } h \geq 2
\end{array}\right.
$$

Given the calculations above, the predictions for round 2 are simple. Because 
$X^{*}(1,3)=B$ and $X^{*}(2,3)=C, X^{*}(0,2)=B$ and $X^{*}(2,2)=A$. Note that delay is costly, and further that delay is costlier (relatively speaking) in later rounds, when there is less to gain from the resultant information. Therefore, since we know from above that $X^{*}(2,4)=A$, it must also be true that $X^{*}(1,2)=A$ :

$$
X^{*}(h, 2)=\left\{\begin{array}{l}
B \text { for } h=0 \\
A \text { for } h=1 \\
C \text { for } h=2
\end{array}\right.
$$

The only calculation remaining is that for round 1. Again, we will simplify the analysis through the use of statement 6 , and begin by comparing $\pi_{A}(0,1)$ and $\pi_{B}(0,1)$ :

$$
\begin{aligned}
\pi_{A}(0,1) & =1+P_{H} \times \pi_{A}(1,2)+\left(1-P_{H}\right) \times \pi_{B}(0,2) \\
& =1+\frac{4}{9} \times 15+\frac{5}{9} \times(8-1) \times 2=\frac{139}{9} \approx 15.44 . \\
\pi_{B}(0,1) & =2 \times(9-1)=16 .
\end{aligned}
$$

Here we have calculated $\pi_{A}(1,2)=1+(0.5 \times 2+0.5 \times 2 / 3 \times 4) \times(8-2)=15$. Hence,

$$
X^{*}(h, 1)=\left\{\begin{array}{l}
B \text { for } h=0 \\
C \text { for } h=1
\end{array} .\right.
$$

We have characterized the entire decision space. $C \in X^{*}(h, t)$ if $h \geq l$ and $B \in X^{*}(h, t)$ if $h \leq l$, except for $(h, t)=(1,2)$ or $(2,4)$, where $X^{*}(h, t)=A$.

Importantly, $B N E$ predicts that subjects in treatments 1 and 2 adopt immediately according to private information, adopting technology $B$ following a private signal of $L$ and technology $C$ following a private signal of $H$. The ex ante expected payoff of this strategy is $\left(0.5 \times 2+0.5 \times \frac{2}{3} \times 4\right) \times(9-1)=\frac{56}{3} \approx 18.67$. A complete characterization of the optimal decision rule calls for $X^{*}$ to include $B$ if $h \leq l$ and $C$ if $h \geq l$, except when $X^{*}(1,2)=X^{*}(2,4)=A$.

\subsection{Treatment $C O$}

Characterization of optimal behavior by subjects who participate in treatment $C O$ is complicated by the possibility of strategic interaction. In this treatment, 
when a subject delays adoption in treatment $C O$, she not only draws an additional private signal, but also can observe prior adoptions of $B$ and $C$ made by fellow subjects as well as the number who opt to remain with the status quo $A$. The value of these observations to a subject turns on her ability to infer the private signals that induced the adoptions. Subjects who adopt in earlier rounds effectively confer an informational externality on later adopters, with no means to internalize the benefits. Consequently, too many subjects may delay adoption in order to gain this information, and consequently too little social learning takes place as subjects decline to bear the cost of informing the others through their early adoption decisions. The externality creates an interdependence in subjects' payoffs for which we need to apply methods of game theory to find an equilibrium.

We begin by asking whether obeying the decision rule constructed for treatments $P$ and $P O$ form an equilibrium when adoption decisions are publicly announced. Specifically, suppose that all round 1 subjects adopt $B$ when they draw $L$, and $C$ when they draw $H$. Can one of the subjects do better by delaying adoption in round 1 regardless of which signal she receives, and then making the best adoption decision in round 2 after drawing a second signal? Because all other subjects implement the optimal decision rule, a subject who delays effectively observes six additional private signals. We will show that this deviation is profitable, so that it cannot be a Bayesian-Nash equilibrium for all subjects to adopt in round 1 by following their private signals.

The deviating individual would choose option $A$ in the first round and view the equivalent of six additional signals. There are 7 different situations that the deviating subject could find herself in in round $2(h=0 \ldots 6)$ if the private signal is $L$, or $(h=1 \ldots 7)$ if the private signal is $H$. The subject's profit-maximizing option after viewing the signal will be to choose either option $B$ or $C$ in round $2{ }^{6}$ Therefore, the deviating player's continuation strategy for round 2 will be to adopt technology $C$ in round 2 if $h \geq 4$, and technology $B$ if $h<4$.

Calculation of the expected value of remaining with technology $A$ requires the determination of the probability of each number of additional $H$ draws, the technology that they would adopt in each case, and its expected value. Assume, for example, that a player's private signal is $L$. The probability that each other subject in the group receives a signal of $L$, and therefore adopts technology $B$, is $\frac{2}{3}$.

\footnotetext{
${ }^{6}$ Essentially, deviating subjects view seven signals. Because this is an odd number of signals, $h-l \neq 0$ and subjects will adopt with certainty in round 2 . We know this since by construction all other subjects have already adopted, so it is identical to a treatment $P$ problem — where it is never profitable to delay adoption once there is a signal imbalance.
} 
Therefore, the probability that a subject learns the outcome of $i$ additional $H$ 's is

$$
\operatorname{Pr}\left(h_{a}=i\right)=\frac{6 !}{i ! \times(6-i) !} \times \frac{4}{9}^{i} \times \frac{5}{9}^{(6-i)} .
$$

Subjects will adopt technology $B$ if there are three or fewer $H$ draws, but technology $C$ if there are four or more $H$ draws. Of course, the expected value of adopting $C$ will depend on the number of $H$ signals. Therefore, the number of $H$ 's affects not only the probability that a subject adopts technology $C$, but also $C$ 's expected value. The benefit of remaining with technology $A$ must account for this:

$\pi_{A}(0,1)=1+\left(2 \times \sum_{i=0}^{3} \operatorname{Pr}\left(h_{a}=i\right)+4 \times \sum_{i=4}^{6} \operatorname{Pr}\left(h_{a}=i\right) \times \frac{2^{i}}{2^{i}+2^{7-i}}\right) \times 7 \approx 19.24$,

and $V_{B}(0,1)=2 \times 8=16$.

As $\pi_{A}(0,1)>\pi_{B}(0,1)$ according to this strategy profile, adopting "honestly" in the first round is not an equilibrium choice in treatment $C O$. Further, for the same strategy profile, $21.73=\pi_{A}(1,1)>\pi_{C}(1,1)=21.33$. This result rules out an equilibrium in which $X^{*}(1,1)=C$ and $X^{*}(0,1)=A .^{7}$

Thus, in treatment $C O$, there can be no equilibrium in which all subjects adopt with certainty in the first round. The information revealed in the decisions of others would be too valuable for subjects to profitably follow such a strategy. There is generally more motivation to delay adoption in treatment $C O$, as delay yields more information.

\section{Results}

We now analyze the outcome of our laboratory experiments, focusing on the evidence that would accept or reject the presence of private learning, social learning, and Bayesian learning (as defined previously). Comparing adoption decisions made in treatments $P$ and $P O$, we find that subjects do tend to be guided by their private signals. However, we also find that observation of earlier adoption decisions tends to improve subject's performance by inducing others to respond to their private signals earlier, even if their decisions are not based on common payoffs.

\footnotetext{
${ }^{7}$ This strategy profile reveal all subjects' signals, making it a profitable deviation to wait and observe other subjects' choices.
} 


\subsection{Private Learning}

Private learning occurs when subjects take into account the signals they receive in their choice of a technology. Delay in that choice recognizes the potential value of private learning. The cleanest test of private learning, therefore, arises with treatment $P$, because in that case subjects' decision problem is not confounded with other sources of information, real or imagined. Evidence of private learning occurs when adoption of technology $B$ decreases with increases in $h$, and that for $C$ increases. Figure 1 compares adoption frequencies of the two technologies for round 1 private signals. Clearly, private learning occurs in round 1 , as adoption frequencies are lower for technology $B$ and higher for technology $C$ for $h=1$ than for $h=0$. Across all subjects in treatment $P, 38.2$ percent adopted technology $B$ when they observed $L$ while 14.1 percent adopted $C$; in comparison, 22.9 percent adopted $B$ and 31.7 percent adopted $C$ when a $H$ signal is observed.

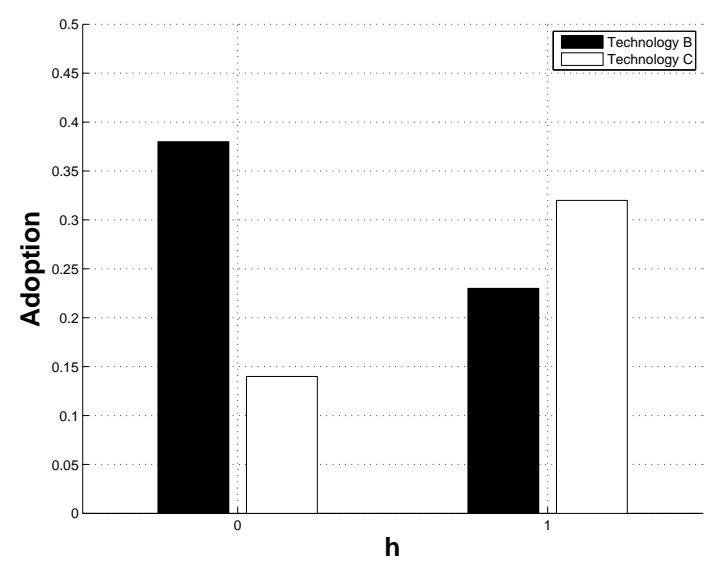

Figure 1: Treatment $P$ Round 1

This leaves 46.5 percent of the subjects opting to wait at least one more round. Some of those subjects decline to adopt in round 2 as well, with 42.0 percent remaining active at least to round 3 . Figure 2 plots the adoption decisions of active subjects in treatment $P$, for rounds 2 and 3 , against $h$.

Private learning clearly occurs in treatment $P$, insofar as the relative tendency to adopt technology $B$ decreases as $h$ increases. Exceptions arise when the private signals skew strongly toward one technology, for example when $h=0$ or $h=3$ in round 3. Selection among subjects is one place to look for an explanation of this anomaly. Note that subjects who viewed $h=3$ in round 3 must have chosen to delay adoption in round 2 with $h=2$. Subjects less receptive to learning from 


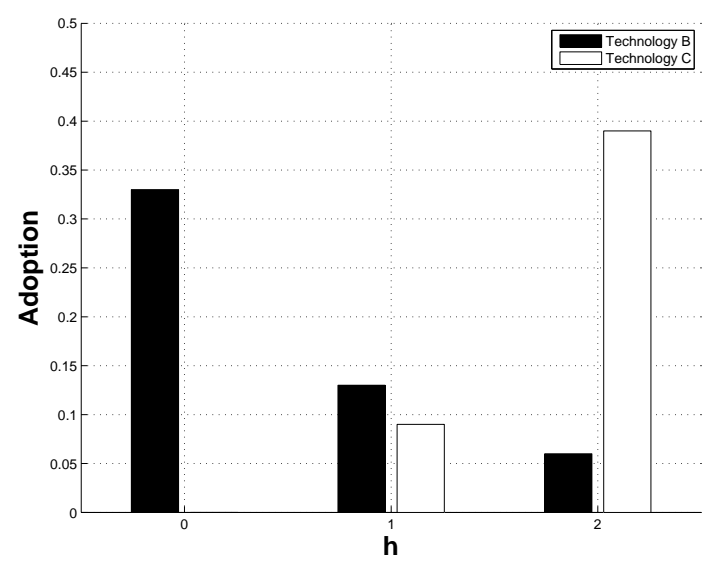

(a) Round 2

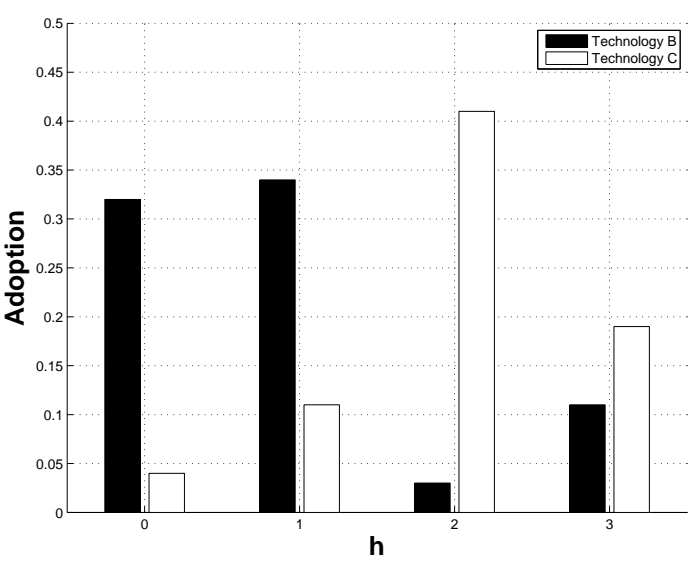

(b) Round 3

Figure 2: Treatment $P$

their private signals are more likely to delay in this way, leaving only such subjects active with $h=3$ in round 3 .

Private learning in treatments $P O$ and $C O$ is more difficult to isolate, since subjects can also take account of prior adoption decisions of other subjects, even when those decisions are not based on common payoffs. In the first round, however, subjects have only their private signal on which to base their decision. Figure 3 plots the round 1 adoption decisions for treatments $P, P O$, and $C O$, depending on whether the subject observed an $L$ or an $H$.

Figure 3 confirms that subjects' round 1 decisions approximated optimal behavior in all three treatments. Subjects adopted technology $B$ with a lower frequency, and $C$ with a higher frequency, when an $H$ was observed.

As discussed above, subjects in round 3 with $h=0$ or $h=3$ necessarily delayed their decision in round 2 with $h=0$ or $h=2$. Therefore, round 3 responses to $h=0$ or $h=3$ may not represent typical preferences of the entire subject pool; that is there is a selection bias concerning whom we observe in round 3 . More generally, beyond the first round, decisions observed in any round represent the preferences of subjects who choose to delay adoption to that particular round. Many reasons to delay the adoption decision can be offered, including riskaversion (reluctance to adopt technology $C$ ), a desire to observe decisions of others (in treatments $P O$ and $C O$ ), or insensitivity to or mistrust of private signals. Similarly, although the information available to subjects in round 1 is identical across 


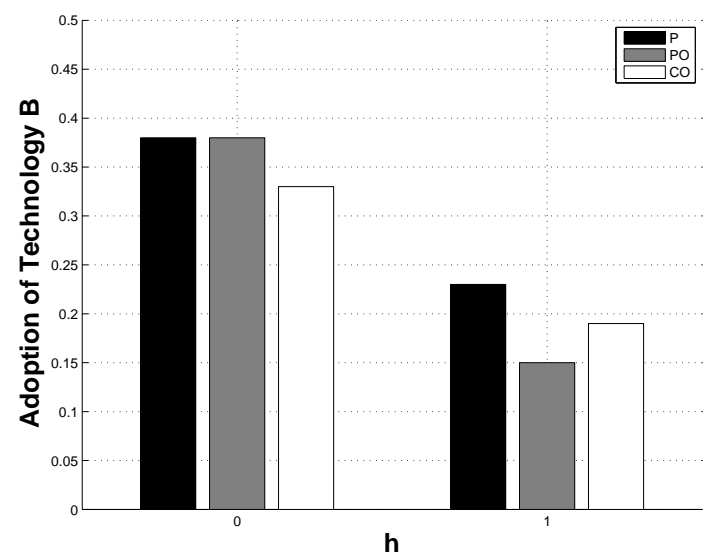

(a) Technology B

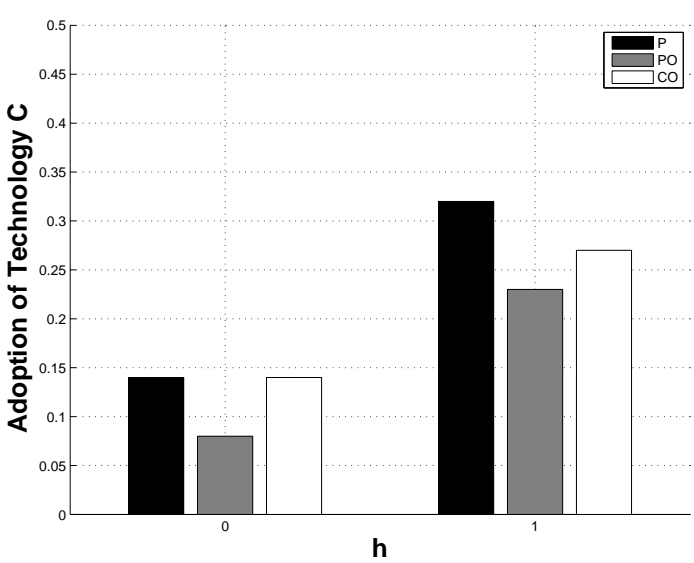

(b) Technology C

Figure 3: Round 1

treatments, round 1 decisions are not. The nature of learning that a subject gains from delaying beyond round 1 differs across the three treatments, as delay will result in different information sets in round 2.

\subsection{Social Learning}

The first possible occurrence of social learning arises in round 2 of treatment $P O$, after subjects have observed any adoption by other subjects in round 1 . Those decisions convey no material information, however, because the payoffs across subjects are uncorrelated. Consequently, any effect the observation may have on subsequent adoption decisions is not social learning in the sense of Bayesian updating but rather a form of social influence. If round 2 adoption rates differ between treatments $P$ and $P O$, then the cause for this difference cannot be Bayesian updating.

Figure 4 shows the adoption rates that were recorded for technologies $B$ and $C$ in treatments $P$ and $P O$, plotted against values of $h$. There are two plots on each axis for treatment $P O$, partitioning adoption decisions by the popular technology. Technology $B$ is dubbed the popular technology when a subject observes more of the other subjects adopting technology B in the first round, and technology $C$ if the opposite is true. The bar labeled $P O B$ shows the fraction of active subjects adopting in treatment $P O$ when technology $B$ is popular, while $P O C$ refers to adoption when technology $C$ is popular. 
Rationality suggests identically declining adoption rates in Figure 4(a) and identically increasing rates in Figure 4(b). In other words, there is no reason for the observation of others to influence decisions. If subjects are inclined to imitate earlier adopters, on the other hand, we would expect the $P O B$ bar to be higher than the $P O C$ bar in Figure 4(a), the opposite in Figure 4(b).

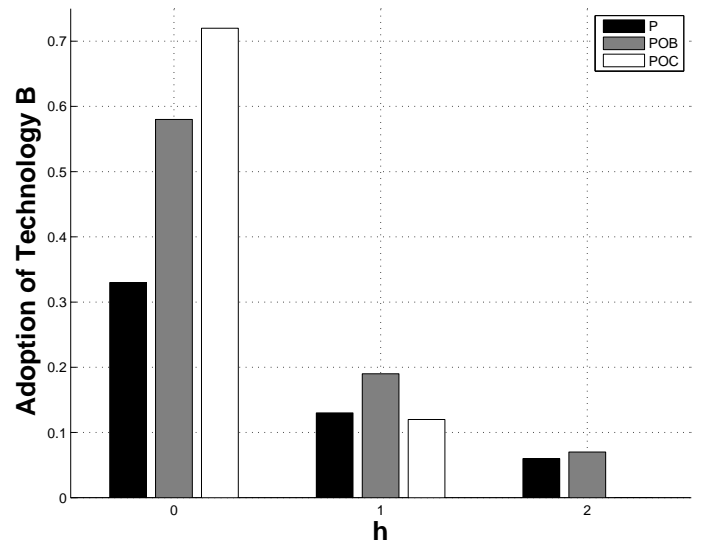

(a) Fraction of Active Subjects Adopting Technology B

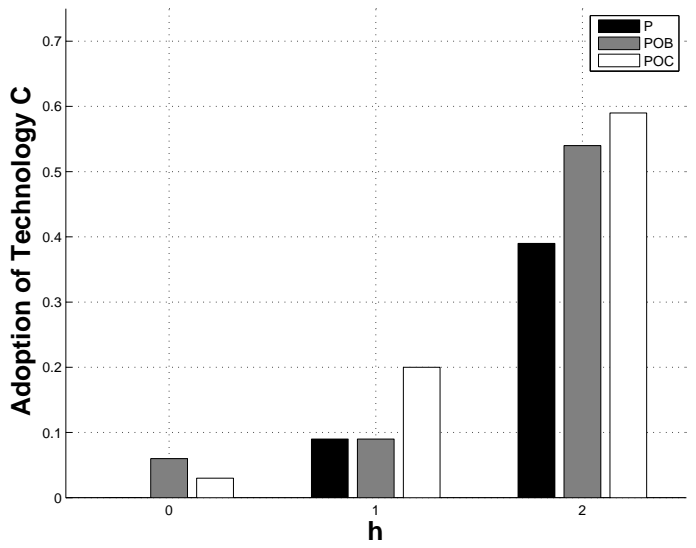

(b) Fraction of Active Subjects Adopting Technology C

Figure 4: Round 2 Adoption by Popularity: Treatments $P$ and $P O$

In some cases, the popular technology is more frequently adopted: the fraction adopting technology $C$ (that is, $P O C$ ) is slightly higher than the fraction for $B$ in treatment $P O$ in Figure 4(b), for example. This is not a robust regularity, however. For example, when $h=0$, subjects adopt technology $B$ more frequently when technology $C$ is popular than when technology $B$ is popular.

A striking regularity emerges from Figure 4 that is not related to the adoption of the popular standard per se. We find a higher level of adoption of either technology in treatment $P O$, no matter which technology is popular. Consider the $h=0$ case within Figure 4(a). Subjects in treatment $P$ adopted technology $B$ (which has an expected value in this case of 2.5 times that of technology $C$ ) only 33 percent of the time. In treatment $P O$, this frequency increases to 72 percent when $B$ is the unpopular technology. It appears that subjects are influenced by the ability to observe their peers, but they appear not to be inclined to take the same actions as the earlier adopters.

The incidence of social learning in round 3 shares one characteristic with the pattern found in round 2: subjects adopt both popular and unpopular technologies 


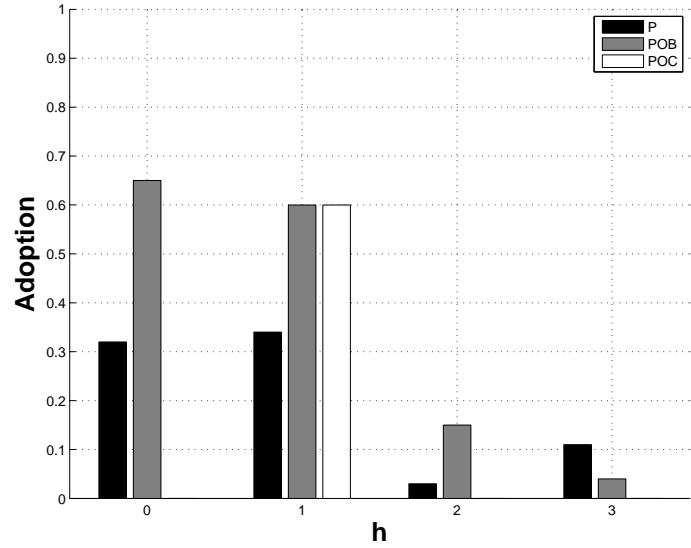

(a) Fraction of Active Subjects Adopting Technology B

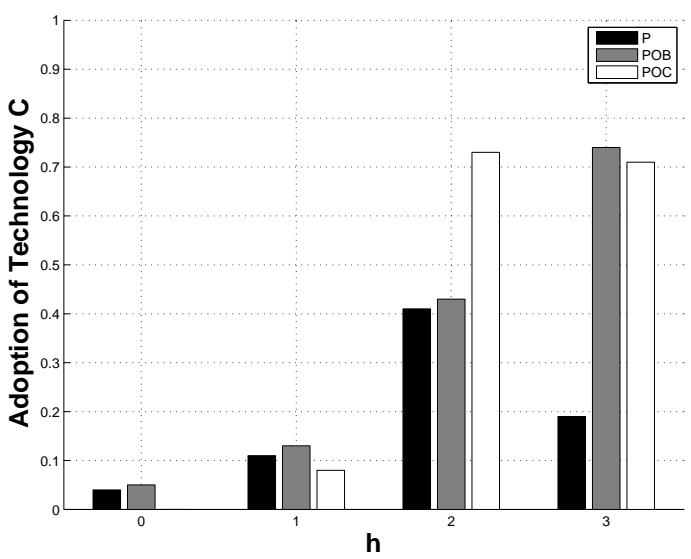

(b) Fraction of Active Subjects Adopting Technology C

Figure 5: Round 3 Adoption by Popularity: Treatments $P$ and $P O$

with a higher frequency when they can observe their peers. Further, anomalous behavior is prevalent for $h=0$ and $h=3$, likely caused by the same selection issues discussed above. An additional pattern emerges in round 3, however. Subjects are more likely to adopt the popular technology in round 3. This suggests another level of selection in our design. Subjects who delay decisions until round 3 appear to be more influenced by the decisions of others than the early adopters.

\subsection{Bayesian Learning}

In treatment $C O$, it is rational for subjects to take into account the observed adoption by other subjects. As adoption decisions are perfectly correlated across subjects, Bayesian updating can take place. Above we found that subjects behave differently in treatment $P O$ than in treatment $P$, and we interpreted this pattern as evidence of social learning. As we discussed, however, any influence that observation has in treatment $P O$ cannot be considered Bayesian learning. Of the three treatments, Bayesian learning can take place only in treatment $C O$. In this section, we examine whether the experimental outcomes confirm that subjects are affected differently by peers' decisions in treatment $C O$ than in treatment $P O$.

Surprisingly, the effect of peer observation is very similar in treatments $C O$ and $P O$, suggesting that Bayesian updating is not the driving force behind this social influence. Subjects appear more sensitive to their private information when they 


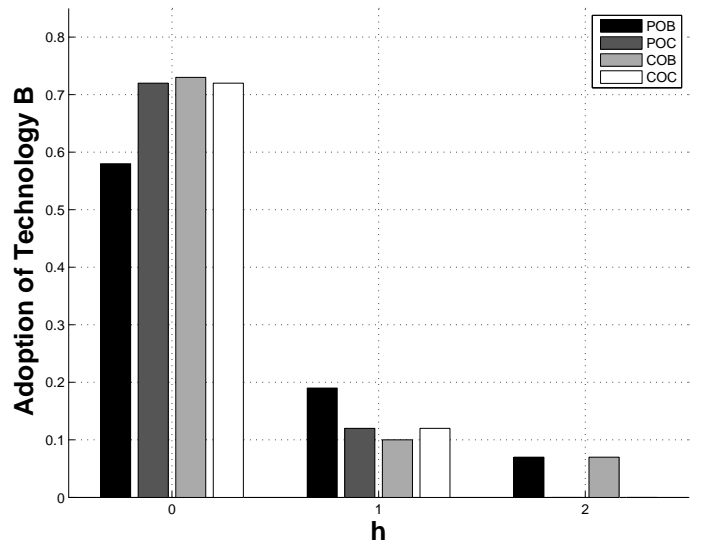

(a) Fraction of Active Subjects Adopting Technology B

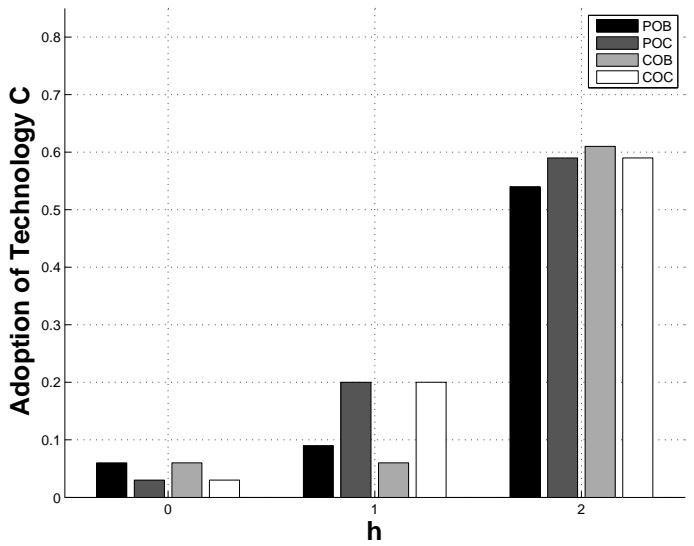

(b) Fraction of Active Subjects Adopting Technology C

Figure 6: Round 2 Adoption by Popularity: Treatments $P O$ and $C O$

can observe the actions of others, regardless of whether this influence is payoff relevant or not.

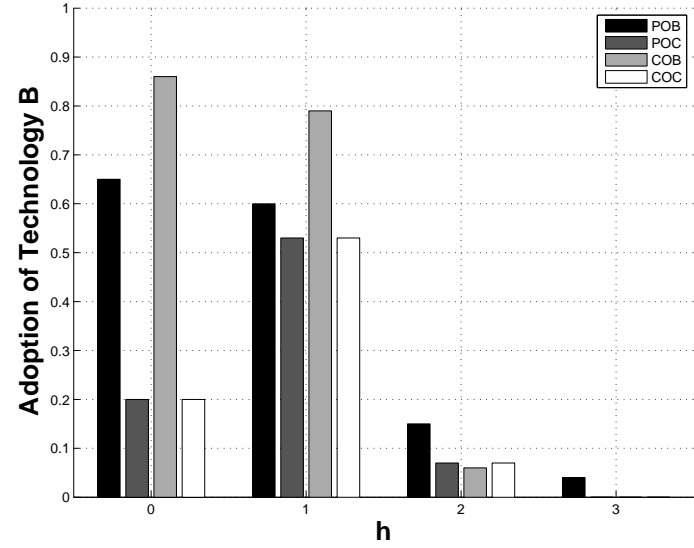

(a) Fraction of Active Subjects Adopting Technology B

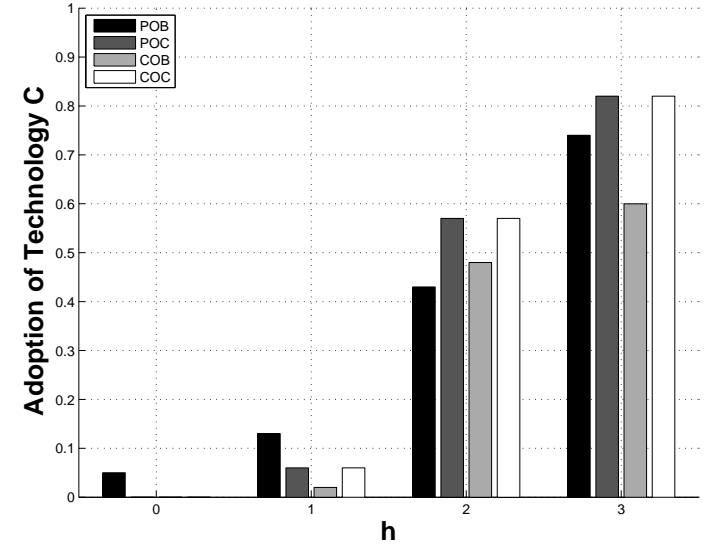

(b) Fraction of Active Subjects Adopting Technology C

Figure 7: Round 3 Adoption by Popularity: Treatments $P O$ and $C O$

We previously uncovered evidence that social learning is more prevalent among subjects who delayed their adoption than among early adopters. Figure 7 suggests that this may also be the case with Bayesian learning. While adoption decisions in 
treatments $P O$ and $C O$ were very similar in round 2, they were different in round 3. Figure 7(a) shows that subjects are more likely to adopt a popular technology $B$ in round 3 in treatment $C O$ than in treatment $P O$. Figure $7(\mathrm{~b})$ shows the same for technology $C$.

\subsection{Choice of Technology}

We now turn to more general comparisons across treatments. Table 1 shows the percentages of subjects who ultimately adopt technologies $B$ and $C$ in each treatment. The differences across treatments are not significant, although subjects were marginally more likely to adopt the risky technology, $C$, when they did not observe other adoptions.

\begin{tabular}{||c||c||c||}
\hline \multicolumn{1}{|c||}{} & \multicolumn{2}{c|}{ Technology Chosen } \\
\hline Treatment & $\mathrm{B}$ & $\mathrm{C}$ \\
\hline$P$ & 51.2 & 48.2 \\
$P O$ & 55.6 & 44.0 \\
$C O$ & 52.5 & 47.3 \\
\hline
\end{tabular}

Table 1: Ultimate Technology Choice by Treatment

Table 2 decomposes adoption decisions into cases where $\pi_{C}=0$ and those where $\pi_{C}=4$. This enables separate evaluation of the decision processes based on which is the more profitable technology.

\begin{tabular}{||c||c|c||c||c||}
\hline \multicolumn{1}{||c||}{} & \multicolumn{3}{c||}{ Technology Chosen } \\
\hline \multicolumn{1}{|||}{} & \multicolumn{2}{c||}{$\pi_{C}=4$} & \multicolumn{2}{c||}{$\pi_{C}=0$} \\
\hline Treatment & $\mathrm{B}$ & $\mathrm{C}$ & $\mathrm{B}$ & $\mathrm{C}$ \\
\hline$P$ & 41.9 & 57.4 & 61.2 & 38.3 \\
$P O$ & 40.8 & 58.5 & 70.8 & 29.0 \\
$C O$ & 41.8 & 58.2 & 65.2 & 34.5 \\
\hline
\end{tabular}

Table 2: Ultimate Technology Choice by Treatment

Looking across treatments, subjects choose the high-payoff technology in roughly six out of 10 rounds. They tend to be slightly more successful using this decision rule when $B$ is the high-payoff technology. In fact, subjects achieve roughly the same measure of success across treatments when the risky technology, $C$ is the high-payoff technology. Notably, when $\pi_{C}=0$, they are most successful in treatment $P O$. This is somewhat surprising, given that subjects presumably have more information on which to base their decisions in treatment $C O$. 


\subsection{Timing of Adoption Decisions}

The theoretical predictions for treatments $P$ and $P O$ involve subjects adopting technology $B$ or $C$ immediately according to their private signal: adopt $C$ if $s_{1}=H$ and adopt $B$ if $s_{1}=L$. Predictions for treatment $C O$ are less clear. Recall that it is not an equilibrium for subjects to choose immediately in treatment $C O$. Consequently, one might expect subjects to come to a decision earlier in treatments $P$ and $P O$. In any case, the timing of adoption decisions is a useful metric to compare subjects' performance across treatments.

Figure 8 shows the hazard rates $^{8}$ across treatments for each treatment. Immediate adoption (adoption in round 1) is clearly more prevalent in treatment $P$, where observation does not take place. Conditional on reaching subsequent rounds, however, adoption is more likely when subjects observe their peers. Figure

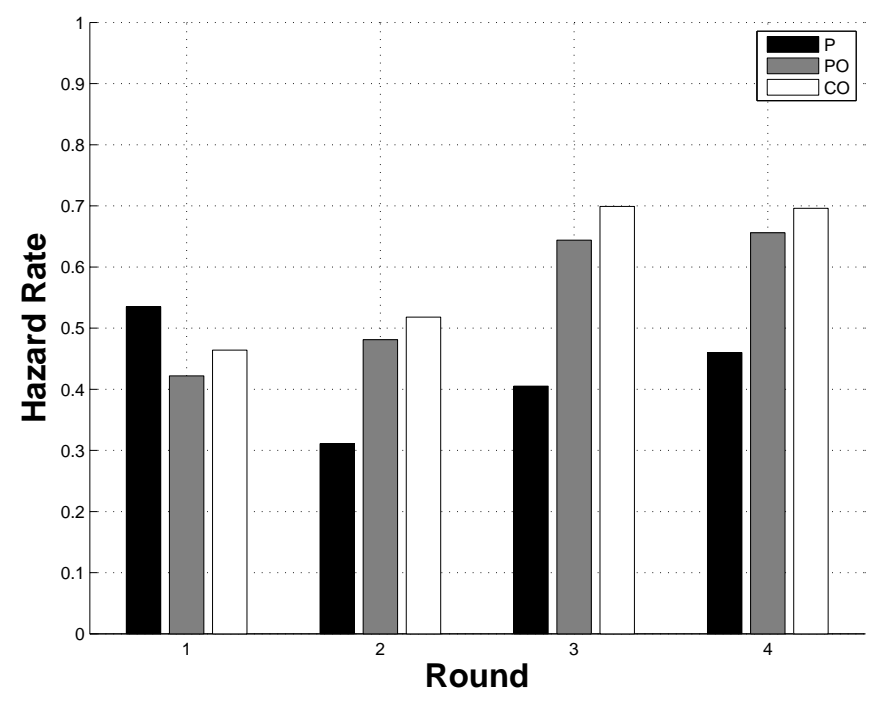

Figure 8: Hazard Rates Across Treatments

9 shows the proportion of subjects in each treatment who have already made their adoption decision. It shows that subjects tend to reach their adoption decisions earlier in treatments $P O$ and $C O$.

\subsection{Profits}

In our experiments, subjects have two motivations. They are incentivized to adopt the high-payoff technology, and to do so quickly. Section 4.4 found few differences

\footnotetext{
${ }^{8}$ The hazard rate is the percentage of subjects adopting option $B$ or $C$, conditional on not adopting before the given round.
} 


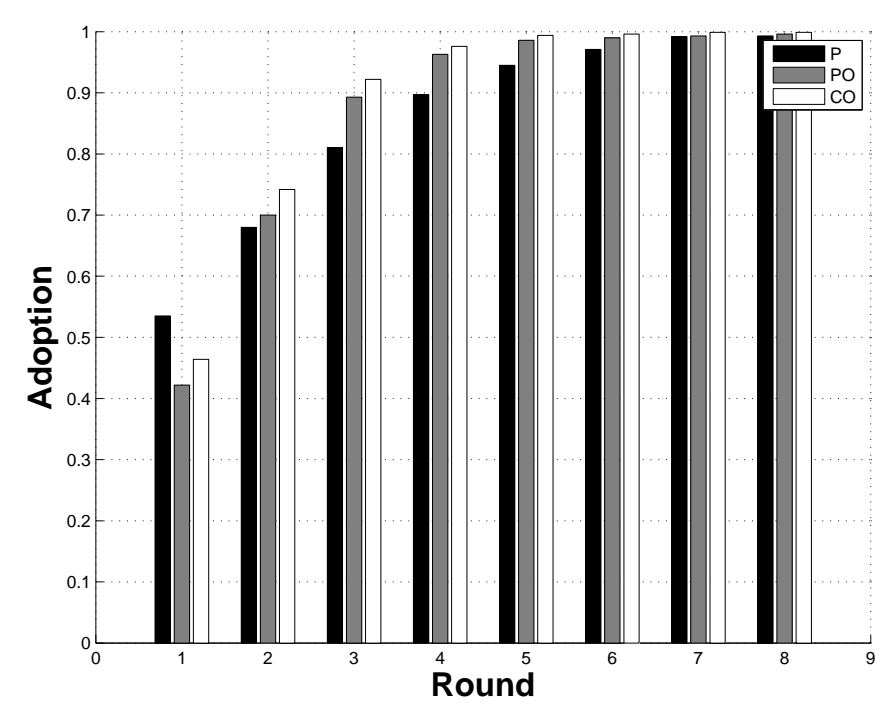

Figure 9: Decision Rates Across Treatments

across treatments in terms of final adoption decisions. Section 4.5 shows subjects in treatment $P$ are more likely to adopt immediately, but also spend more time overall in technology $A$.

Finally we turn to the hypothesis that subjects attempt to maximize their profits, so profit is a useful measure of subject performance. Table 3 compares average profits across treatments. Note that observation tends to help subjects earn higher payoffs, even when payoffs are uncorrelated. Although these differences are insignificant at the 5 percent level, they provide anecdotal evidence that the observation of one's peers improves performance in our decision problem.

One explanation for this regularity can be found in the earlier overall adoption rates in treatments $P O$ and $C O$. Our results suggest that, for a certain class of decision problems, the observation of one's peers may improve performance by causing some subjects to follow their private information earlier, even if the decisions that they observe are uncorrelated to their own.

\begin{tabular}{||c||c||c||c||}
\hline Treatment (Obs.) & Profit (std. dev) & Profit for $V=0$ (std. dev) & Profit for $V_{C}=4$ (std. dev) \\
\hline$P$ & $16.5(9.7)$ & $9.7(6.7)$ & $22.8(7.5)$ \\
$P O$ & $17.0(9.0)$ & $11.1(6.4)$ & $22.8(7.2)$ \\
$C O$ & $17.2(9.6)$ & $10.0(6.8)$ & $23.2(7.1)$ \\
\hline
\end{tabular}

Table 3: Profits by Treatment 


\section{Conclusion}

We explore the timing and selection of innovative technologies using laboratory experiments that isolate the roles of private information and social learning. Implementing three treatments that are nested by the amount of information made available to subjects, we are able to gauge the extent to which that information is incorporated into private and social technology adoption. Specifically, we find that roughly half of all subjects do not follow the theoretical prescription to adopt the technology favored by their first private signal, but instead they delay adoption with the apparent intention of acquiring additional information. With social information available (in this case knowing the decisions of others, whether this is payoff-relevant or not), subjects are slightly less likely to choose in the first round but more likely to choose thereafter. Since on average they choose earlier overall, their profits are higher, although they are not significantly more likely to choose the optimal new technology. This suggests that people pay attention to others even if others' choices should not affect them, and that relatively speaking they pay more attention to the fact that others do something than to what in particular others do. This has natural implications both for firms that are attempting to maximize profits and for those that are attempting to predict what choices firms will make. A natural extension to this work is to additionally include the possibility of direct network payoff externalities. In a companion paper - Jamison, Owens, and Woroch (2008) — we report on the results of a laboratory experiment with that feature. 


\section{References}

[1] Aalbers, Rob, Eline van der Heijden, Jan Potters, Daan van Soest, and Herman Vollebergh. (2007). Technology adoption subsidies: An experiment with managers. Tinbergen Institute Discussion Paper 07-082/3.

[2] Anderson, Lisa R. and Charles A. Holt. (1997). Information cascades in the laboratory, American Economic Review, 87(5), 847-862.

[3] Banerjee, Abhijit (1992). A simple model of herd behavior," Quarterly Journal of Economics, 107(3), 797-817.

[4] Bikchandani, Sushil, David Hirschleifer and Ivo Welch. (1992). A theory of fads, fashion, custom and cultural change as informational cascade. Journal of Political Economy, 100(5), 992-1026.

[5] Çelen, Boğaçhan and Shachar Kariv. (2004). Distinguishing informational cascades from herd behavior in the laboratory. American Economic Review, 94(3), 484-497.

[6] Chamley, Christopher. (2004). Delays and equilibria with large and small information in social learning. European Economics Review, 48(3), 477-501.

[7] Chamley, Christopher, and Douglas Gale. (1994). Information revelation and strategic delay in a model of investment. Econometrica, 62(5), 1065-1085.

[8] Drehman, Mathias, Jörg Oechssler, and Andreas Roider. (2007). Herding with and without payoff externalities - an internet experiment. International Journal of Industrial Organization, 25, 391-415.

[9] Ellison, Glenn and Drew Fudenberg. (1993). Rules of thumb for social learning. Journal of Political Economy, 101(4), 612-43.

[10] Geroski, Paul. (2000). Models of technology diffusion. Research Policy, 29(45), 603-625.

[11] Griliches, Zvi. (1957). Hybrid corn: An exploration in the economics of technological change. Econometrica, 25, 501-522.

[12] Isaac, Mark and Stanley Reynolds (1992). Schumpeterian competition in experimental markets. Journal of Economic Behavior \&S Organization, 17(1), 59-100. 
[13] Isaac, Mark and Stanley Reynolds. (1988). Appropriability and market structure in a stochastic invention model. Quarterly Journal of Economics, 103(4), 647-671.

[14] Jamison, Julian, David Owens, and Glenn Woroch. (2008). Social learning with endogenous decision timing and payoff externalities: experimental tests of network externalities. Working paper, Dec. 26, 2008.

[15] Jensen, Richard. (1982). Adoption and diffusion of an innovation of uncertain profitability. Journal of Economic Theory, 27(1), 182-193.

[16] Jensen, Richard. (1988). Information capacity and innovation adoption. International Journal of Industrial Organization, 6(3), 335-50.

[17] Kapur, Sandeep. (1995). Technology diffusion with social learning. Journal of Industrial Economics, XLIII(2), 173-199.

[18] Mansfield. (1961). Technical change and the rate of imitation. Econometrica, $29(4), 741-766$.

[19] Rogers, Everett. (1962). The diffusion of innovations. New York: Free Press.

[20] Sgroi, Daniel. (2003). The right choice at the right time: A herding experiment in endogenous time. Experimental Economics, 6, 159-180.

[21] Zhang, J. B. (1997). Strategic delay and the onset of investment cascades. Rand Journal of Economics, 28(1), 188-205.

[22] Zizzo, Daniel John (2002), "Racing with uncertainty: a patent race experiment. International Journal of Industrial Organization, 20, 877-902 


\section{Appendix: Experimental Instructions (treatment $\mathrm{CO}$ ):}

Welcome to this experiment on decision making and thank you for being here. You will be compensated for your participation in the experiment, though the exact amount you will receive will depend on the choices you and others make and on random chance (as explained below). Please pay careful attention to these instructions, as a significant amount of money is at stake.

Information about the choices that you make during the experiment will be kept strictly confidential. Your name will appear only on the payment-receipt form and will not be linked to any specific choices you make. You will not be asked to reveal your identity or the content of your decisions to anyone else (either the experimenter or other participants) at any time during or after the experiment. In order to maintain privacy and confidentiality, please do not speak to anyone during the experiment and please do not discuss your choices with anyone even after the conclusion of the experiment.

The experiment will consist of a series of 16 problems, the first of which will be a practice problem for which you will not be paid. Each problem is called a Decision Sequence and is made up of 8 rounds. During each Decision Sequence, you will decide if and when to move between three alternative courses of action: options A, B, and C. You will begin each Decision Sequence following the default option, A. During round 1 of 8, you will be asked to choose one of three options: to remain with option A, to switch to B, or to switch to $\mathrm{C}$. As long as you remain with $\mathrm{A}$, you will be given the same three options during each subsequent round in the sequence. After you switch to B or C, you will no longer have the opportunity to move between options. You will remain with either B or C (depending on which one you selected) each subsequent round until the end of the sequence. For example, you may choose to remain in option A for the first three rounds, and then switch to option $\mathrm{C}$ in round 4 . You must then remain in option $\mathrm{C}$ for the rest of that Decision Sequence.

At the beginning of each sequence, you will be placed into a group with 5 other participants that are in the lab with you. Group assignments will be made randomly and will change at the end of each sequence. At the beginning of each round, you will be told how many of your group members were following each alternative (A, B, and C) as of the end of the previous round.

There is a payoff associated with following each option. For each round that you follow option A, you will receive 1 token. For each round that you follow B, you will receive 2 tokens. The payoff for $\mathrm{C}$ in each decision sequence is the same for all members of your group and will be randomly determined at the beginning of each decision sequence; it will remain the same until the end of that decision sequence. The payoff for $C$ will either take a value of 0 tokens per round or 4 tokens per round, both being equally likely. You will not be told if your group's payoff for $\mathrm{C}$ is 0 or 4 in any given decision sequence, but at the beginning of each round you will be shown the result of a coin flip that will help you determine what the payoff for $C$ is. If the payoff for $C$ is 0 , the coin flip will result in an $\mathrm{H}$ with $1 / 3$ probability and an $\mathrm{L}$ with $2 / 3$ probability. If the payoff for $\mathrm{C}$ is 4 , the coin 
flip will result in an $\mathrm{H}$ with 2/3 probability and an $\mathrm{L}$ with $1 / 3$ probability. The payoff for $\mathrm{C}$ will be the same for each member of your group in each decision sequence, but the coin flips themselves are generated randomly for each group member individually, and therefore may or may not be the same. Figure 1, below, illustrates the payoffs.

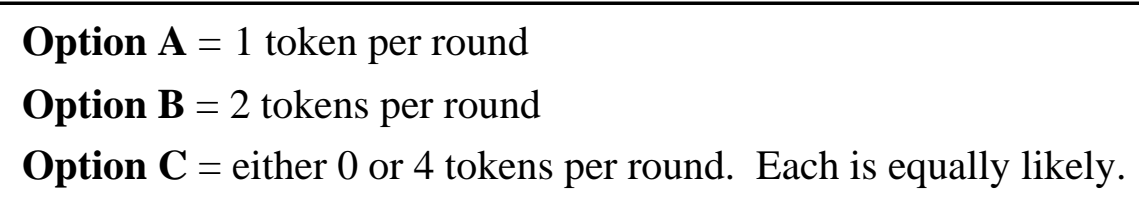

\section{Coin Flips:}
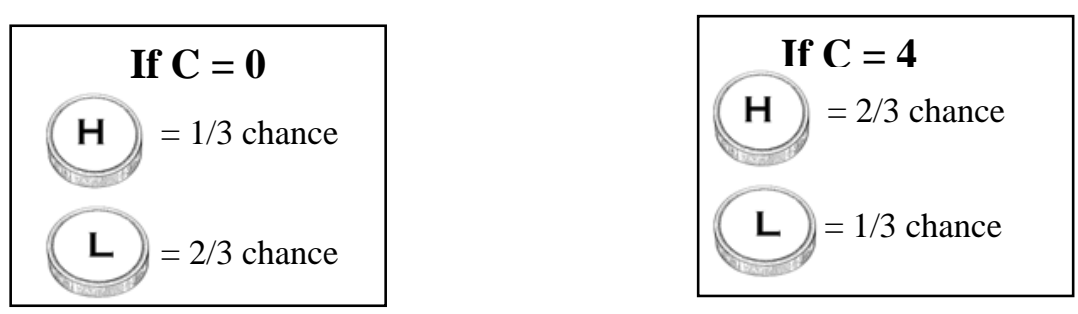

\section{Figure 1. Payoffs for Options A, B, and C.}

Your payoffs for the 8 rounds in a sequence will be tallied at the end of the sequence. For example, if you decided to remain with option A for the first 4 rounds and then switched to option B, you would receive $4 * 1+4 * 2=12$ tokens. Your tokens from all 15 Decision Sequences will be added at the end of the experiment and converted to U.S. dollars (the exchange rate is: 12 tokens $=\$ 1$ ), and you will be given a check for that amount.

The payoff for option C can be 4 or 0 tokens. For example, if you remain with option A for the first two rounds and switch to option $\mathrm{C}$ in the third round, your payoff will be $2 * 1+6 * 4=26$ if the payoff for option $C$ turns out to be 4 , and your payoff will be $2 * 1+6 * 0=2$ if the payoff for $C$ turns out to be 0 . Note that the true value of the payoff for $\mathrm{C}$ will remain the same (either 0 or 4 ) for all 8 rounds of the Decision Sequence.

For each round that you remain with option A, you will view a flip of the coin that will help you determine the payoff for option C. For example, if you choose option A during rounds 1, 2 and 3, you will see four flips of the coin (at the beginning of rounds 1-4).

After all 8 rounds of a Decision Sequence, you will be told how many tokens you accumulated in that sequence. Then a new sequence will begin and you will go through the same process again. Remember that payoff for $\mathrm{C}$ is independently chosen for each sequence, so the value of C's payoff in previous sequences is not an indication of what it will be in future sequences. After all 15 Decision Sequences, your tokens from all sequences will be added together, converted to dollars, and given to you in the form of a check. 


\section{Computer Program Description}

To make your decisions you will use the computer in front of you. Right now, you can see an initial waiting screen. The program will be activated when the instructions are finished.

Once the program is activated, you will be given a short quiz and go through a practice decision sequence to make certain that you correctly understand how the experiment will work. After you have completed the quiz and the practice sequence, the experiment will begin. At that time, a new window will pop up and replace the initial waiting window. The new window will resemble the one shown below. The upper-left of the screen informs you that you are in the first of 15 Decision Sequences. The upper-right of the screen shows how much time you have remaining to make your decision. You will have a total of 20 seconds to make your decision, after which the computer will choose option A for you. The center of the screen will inform you that you are in round 1 and tell you the result of a coin flip that will help you determine the payoff for option C. The screen below shows that the first flip of the coin resulted in an $\mathrm{H}$. Finally, you are asked for your decision. You may choose option A, option B or option C by placing the cursor on the corresponding button and left-clicking.

Decision Sequence

This is round 1 of 8

The result of your first coin flip is $\mathrm{H}$

\begin{tabular}{|c|}
\hline continue with $\mathrm{A}$ \\
\hline switch to $\mathrm{B}$ \\
\hline switch to $\mathrm{C}$ \\
\hline
\end{tabular}


If you choose option B or C in the round 1, you remain with that option for the remainder of the rounds of the Decision Sequence. In this case, you will again see the waiting screen that is in front of you now, which will remain until the other participants are finished with the first Decision Sequence.

If you choose option A in the round 1, you will see a similar screen as the second round begins. Notice that the upper-left of the screen shows that you are still in the first decision sequence. As shown in the upper-right, you will again have 20 seconds to make your decision, after which the computer will select option A for you. The screen will inform you that you are in the round 2. It will also show the options chosen by other members of your group in the previous round. Below, the screen shows that 6 group members chose A, while no group members chose B or C. Just underneath, the screen shows the result of a new coin flip. The screen shown below indicates that the coin flips for rounds 1 and 2 resulted in an $\mathrm{H}$ and an $\mathrm{L}$. Notice that you are shown the results of the coin flips for both round 1 and round 2.

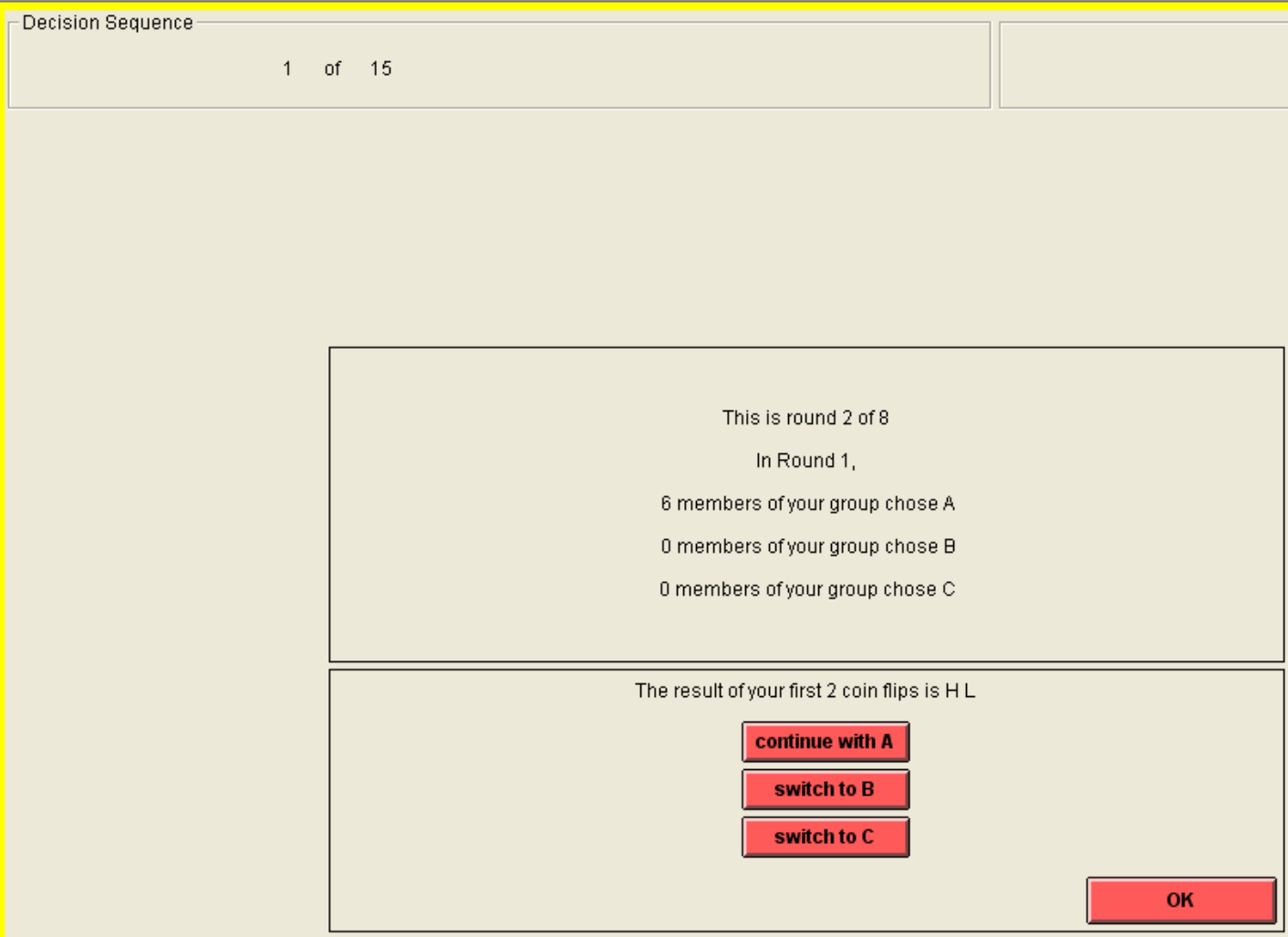


If you choose option B or C in round 2, you remain in that option and are therefore shown the waiting screen. If you choose option A in round 2, you will be told the result of an additional coin flip and be asked to make another decision.

This process will repeat itself until you choose option B or option C, or until you complete round 8. After the Decision Sequence is over, you see the feedback screen. The feedback screen shows you the payoff for C, 0 , and your profit for the round, 1 token.

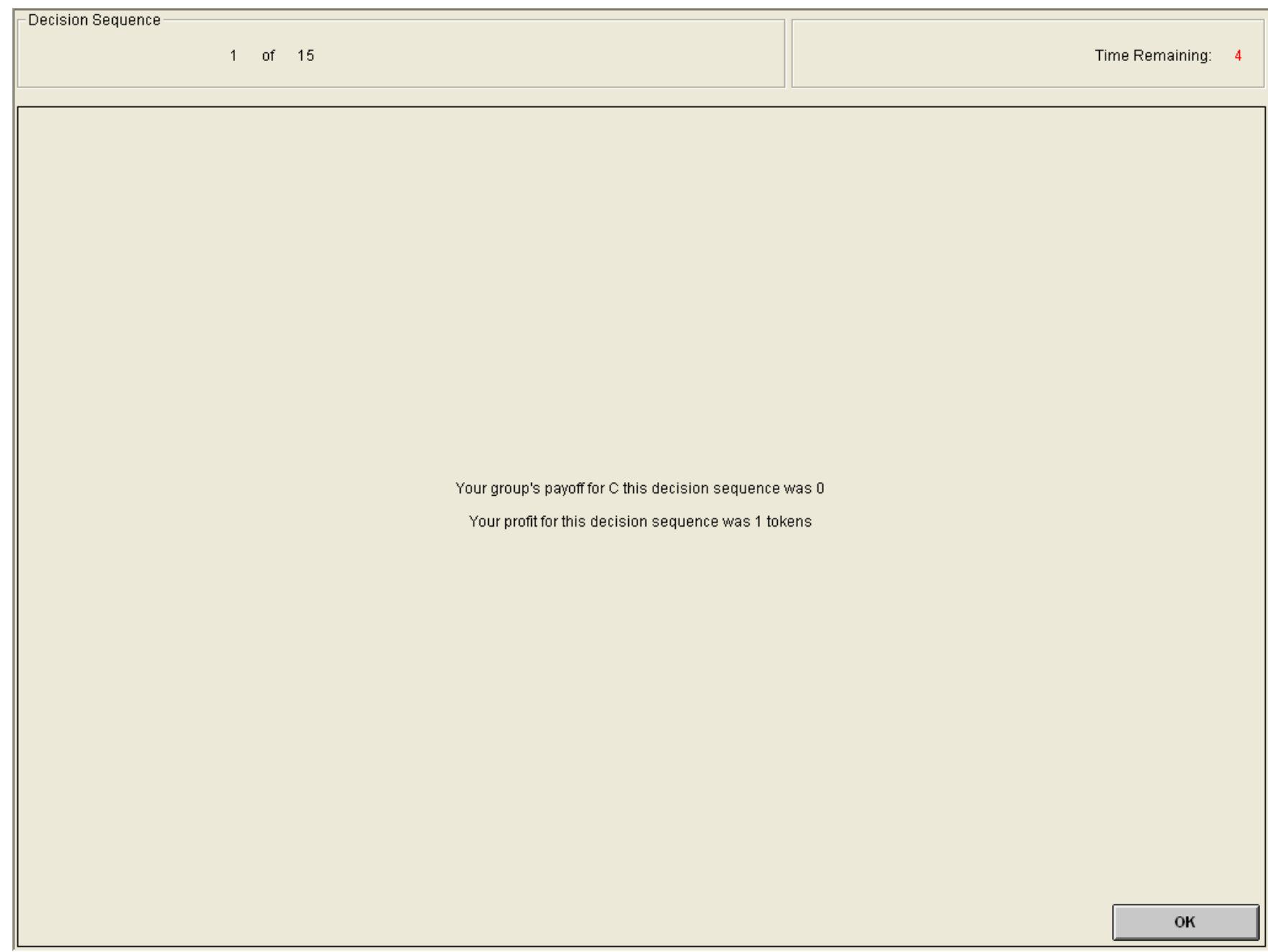

You have a maximum of 20 seconds to observe the feedback screen from the first Decision Sequence. You may click the OK button in the lower right corner of the screen when you are ready to proceed to the next Decision Sequence. After 20 seconds, you will proceed automatically.

Then, the new Decision Sequence will start, and the computer will again randomly place you into a group with 5 other participants, randomly select a coin, show more information and ask for a new decision. 
After the last round is finished, a final screen will pop up, informing you of your total earnings for this experiment.

\section{Rules}

Please do not talk with anyone during the experiment. We ask everyone to remain silent until the end of the last round.

Your participation in the experiment and any information about your earnings will be used solely for research purposes. Your name and association to your decisions will be kept strictly confidential. Your payment receipt and participation form will be the only places in which your name is recorded.

\section{Questions}

Any clarification questions should be asked at this time. Please raise your hand and wait for an instructor to come to your desk. 